\title{
Application of integer programming in project investment
}

\author{
CHUNXUE ZHAO \\ Anyang Normal University \\ School of Mathematics and Statistics \\ Xiange Street 436, Anyang \\ P.R.CHINA
}

Abstract: - Integer programming is widely used to solve optimization problems in economy, management, communication and engineering.In this paper, we use the integer programming to solve the project investment problem, which provides a solution to this type of problem.

Key-Words: - assignment problem, project investment, mathematical model

Received: May 2, 2020. Revised: October 31, 2020. Accepted: November 2, 2020. Published: November 13, 2020.

\section{Introduction}

Project investment is a kind of long-term investment behavior which takes a specific project as the object and is directly related to the new project or the renovation project. How to allocate the limited resources to the unlimited investment of market demand, meet the requirements of project investment allocation and obtain the maximum economic benefits is a problem that every enterprise investment decision maker must solve. There are many methods of investment decision making, and the linear programming theory and mathematical model are effective tools to deal with some kinds of investment decision making problems. In addition, Many practical problems can be transformed into assignment problems, for example, Xiao et.al.[1] studied the application of assignment problems in the supplier selection in supply chain. Liu et.al.[2] discussed the generalized assignment problem and its generalization application within the armaments transportation. $\mathrm{Liu}[3]$ analyzed the multiple attribute group decision making based on the optimal linear assignment. Goldberg et.al.[4] researched the messy genetic algorithms. Cantu-Paz[5] gave a summary of research on parallel genetic algorithms. Eshelman[6] provided the CHC adaptive search algorithm. Srinivas[7] had a research on the adaptive probabilities of crossover and mutations in GAs. Houck et.al.[8] gave a genetic algorithm for function optimization. Tsujimura et.al.[9] provided the genetic algorithms for solving multi-processor scheduling problems.Nakano[10] studied the conventional genetic algorithm for job shop problems. Li et.al.[11] gave uniform approach to model-based fuzzy control system design and structural optimization. In this paper, we first review the concepts of integer programming and the branch and bound method of integer programming, and then we use the branch and bound method to solve the maximum profit problem.

In this paper, we first review the concepts of Linear programming and integer programming. and then we use the integer programming to solve the choice program of project investment.

\section{The Linear Programming and Integer Programming}

Linear programming is a branch of mature in operational research. The maximum or minimum issues in linear objective function under the linear condition are collectively referred to as linear programming problem. Solutions for the feasible are solutions of linear constraints, the set made up of all the feasible solution is called the feasible region. The decision variables, objective function and constraint conditions are the three elements of linear programming problem.

In linear programming problem, the optimal solution could be a fraction or a decimal, but for some specific problems, it can only take the integer variables, such as the number of a factory, the numbers of the running machine. Therefore, we call 
the integer programming for planning problem requiring the part or all of the decision variables to be integer values. The basic expression of integer programming is

$$
\begin{aligned}
& \max (\min ) z=\sum_{j=1}^{n} c_{j} x_{j} \\
& \text { s.t }\left\{\begin{array}{c}
\sum_{j=1}^{n} a_{i j} \leq(=, \geq) b_{j} \quad(i=1,2, \cdots, m) \\
x_{j} \geq 0, x_{j} \text { is an int eger }(j=1,2, \cdots, n)
\end{array}\right.
\end{aligned}
$$

\section{Modeling}

A company has 2.2 billion yuan of capital available for investment, and now there are six investment projects to choose from. The amount of investment required for each project and the estimated annual income are shown in table 1.

Table 1: Investment amount and income of each project(unit: one hundred million yuan).

\begin{tabular}{|c|c|c|c|c|c|c|}
\hline project & 1 & 2 & 3 & 4 & 5 & 6 \\
\hline Investment & 5 & 2 & 6 & 4 & 6 & 8 \\
\hline Income & 0.5 & 0.4 & 0.6 & 0.5 & 0.9 & 1 \\
\hline
\end{tabular}

An investment plan is chosen to maximize returns.This problem involves the selection of project investment and investment issues, each project has two possibilities, which obtain a 0-1 integer programming model, investment can be expressed as 1 , not investment be 0 , then we use the basic expression for solving integer programming. There is 2.2 billion to invest in the company, the investment amount of each project is different, so we put the connection between the investment amount and the constrained variable as the constraints to the problem, the maximum return on investment as objective function to establish the model.

The total amount of investment $I$ satisfies $I=5 x_{1}+2 x_{2}+6 x_{3}+4 x_{4}+6 x_{5}+8 x_{6} \quad$, Expected annual total return on investment $p$ satisfies

$p=0.5 x_{1}+0.4 x_{2}+0.6 x_{3}+0.5 x_{4}+0.9 x_{5}+x_{6}$, the mathematical model is following.

$$
\begin{gathered}
\max p=0.5 x_{1}+0.4 x_{2}+0.6 x_{3}+0.5 x_{4}+0.9 x_{5}+x_{6} \\
\left\{\begin{array}{c}
5 x_{1}+2 x_{2}+6 x_{3}+4 x_{4}+6 x_{5}+8 x_{6} \leq 22 \\
x_{i}=0,1(i=1,2, \cdots, 6)
\end{array}\right.
\end{gathered}
$$

Then the branch and bound method is used to solve the model. Form table 1, we find that there is no obvious rule for the change between the data of investment amount and return, so the calculation can be simplified by building a common standard, that is, the return rate of each project can be obtained, and the following solution can be obtained by unit investment return. Therefore, table 1 is changed to table 2.

Table 2: the return rate(unit: one hundred million yuan).

\begin{tabular}{|l|l|l|l|l|l|l|}
\hline project & 1 & 2 & 3 & 4 & 5 & 6 \\
\hline Investment & 5 & 2 & 6 & 4 & 6 & 8 \\
\hline Income & 0.5 & 0.4 & 0.6 & 0.5 & 0.9 & 1 \\
\hline Return rate & 0.1 & 0.2 & 0.1 & $\begin{array}{l}0.12 \\
5\end{array}$ & 0.15 & $\begin{array}{l}0.12 \\
5\end{array}$ \\
\hline
\end{tabular}

The problem is simplified according to the return rate of table2. Form table 2, the investment rate of the second, fourth, fifth, sixth investment projects is high and they are invested first. Because the return rate of the first project is as low as he third project, so we need to make a choice in the two projects. Then the original problem is transformed into the relaxation problem, two groups optimal solution can be obtained as follows, respectively.

$$
\begin{aligned}
& \left(0,1, \frac{1}{3}, 1,1,1\right)=\left(x_{1}, x_{2}, x_{3}, x_{4}, x_{5}, x_{6}\right) \\
& \left(\frac{2}{5}, 1,0,1,1,1\right)=\left(x_{1}, x_{2}, x_{3}, x_{4}, x_{5}, x_{6}\right)
\end{aligned}
$$

The revenue amount corresponding to these two solutions is 300 million yuan, it is obvious that they are not feasible solutions to the original problem. Therefore, to branch them, branch the first solution, namely, branch the decision variable $x_{3}$ into 0 and 1 , when $x_{3}=0$, the get optimal solution is $\left(\frac{2}{5}, 1,0,1,1,1\right)$. The solution is not a feasible solution to the original problem, so the branch 
continues in the next step. when $x_{3}=1$, the get optimal solution is $(0,1,1,0,1,1)$, the solution is a feasible solution to the original problem, so the solution is retained. At this time, the profit is 290 million yuan. Then $0 \leq p^{*} \leq 2.9 \quad\left(p^{*}\right.$ is the maximum return on investment). we continue to deal with the solution $\left(\frac{2}{5}, 1,0,1,1,1\right)$, in the previous step, that is, branch the decision variable $x_{1}$, and leave the decision variable $x_{3}$ unchanged, we divide it into these two cases: $x_{1}=0$ and $x_{1}=1$. When $x_{1}=0, x_{3}=0$, the other variables remain unchanged, the revenue of the solution is 280 million yuan. When $x_{1}=1, x_{3}=0$, we get two sets of optimal solutions $\left(1,1,0, \frac{1}{4}, 1,1\right)$ and $\left(1,1,0,1, \frac{5}{8}, 1\right)$, the corresponding annual income is 292.5 million yuan. At this time, only the two sets of solutions with corresponding annual income of 292.5 million yuan are considered. They are not feasible solutions to the original problem and $0 \leq p^{*} \leq 2.925$, So keep branching.

Then we branch $x_{4}$ in $\left(1,1,0, \frac{1}{4}, 1,1\right)$, it is branch into two cases: $x_{4}=0$ and $x_{4}=1$, when $x_{1}=0, x_{3}=0, x_{4}=0$, the optimal solution is $(1,1,0,0,1,1)$, the revenue was 280 million yuan, when $x_{1}=1, x_{3}=0, x_{4}=1$, the optimal solution is $\left(1,1,0,1,1, \frac{5}{8}\right)$, the revenue was 292.5 million yuan, continue to branch it.

We branch $x_{6}$ into two cases: $x_{6}=0$ and $x_{6}=1$, when $x_{1}=1, x_{3}=0, x_{4}=1, x_{6}=0$, the optimal solution is $(1,1,0,1,1,0)$, At this point, the revenue is 230 million yuan, when $x_{1}=1, x_{3}=0, x_{4}=1, x_{6}=1$, the optimal solution is $\left(1,1,0,1, \frac{1}{2}, 1\right)$, At this time, the revenue is 275 million yuan, greater than 230 million yuan, but the solution is not a feasible solution to the original problem. In conclusion, $2.75 \leq p^{*} \leq 2.925$, so 290 million yuan is the optimal value of the original problem, At this time, the optimal solution is $(0,1,1,0,1,1)$. The branching process is represented by a tree diagram in figure 1 .

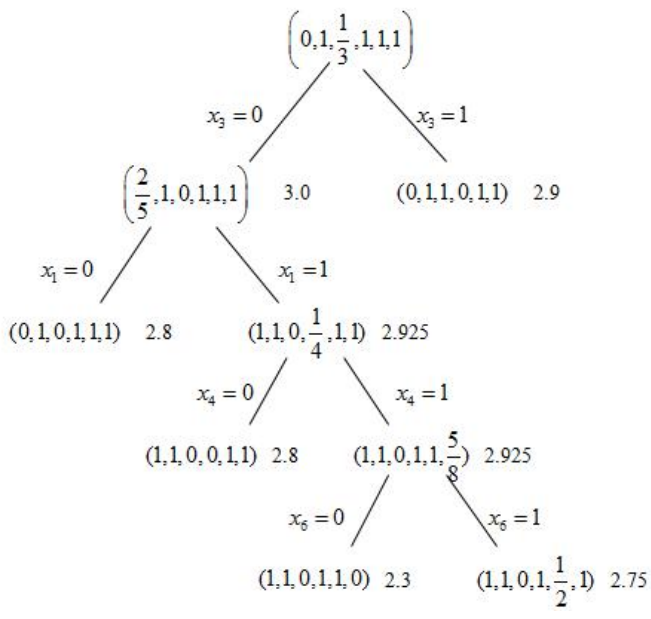

Fig.1 The branching process

To sum up, it can be concluded that the investment in the second, third, fifth and sixth projects yields the most, benefiting 290 million yuan.

Integer programming can also be used for a variety of assignment problem, often encountered such a problem in practical life, there are $x$ tasks need to be completed by $y$ individual, each task has a different character, each person also has the characteristics of different, so different people have different efficiency to complete various tasks, this is about to use integer programming for personnel assigned. In combinatorial optimization problem, the method of using $0-1$ integer programming to find the optimal solution in the set of feasible solution. Also in the economic management problems, can use the integer programming model to solve the problem of investment, such as cutting problem.

\section{Conclusion}

In this paper, we use the integer programming model and the branch and bound method to study the project investment problem, which provides the theoretical support for the solution of such problems.

Acknowledgements: The research was supported by National Natural Science Foundation of China(11801012) and National Social Science Foundation of China(18CTJ007).

\section{References:}

[1] Xiao Zhi, Zhong Bo, Li Yingbing, The application of assignment problems in the 
supplier selection in supply chain, Operations Research and Management Science, 11, 3, 2002, pp. 63-68.

[2] Liu Jiaxue, Chen Shiguo, Generalized assignment problem and its generalization application within the armaments transportation, Mathematics in Practice and Theory, 36, 1, 2006, pp. 199-203.

[3] Liu Jiaxue, The multiple attribute group decision making based on the optimal linear assignment, Systems Engineering, 19, 4, 2001, pp. 32-36.

[4] Goldberg D E, Korb B, Deb K, Messy genetic algorithms: motivation, analysis and first results, Complex Systems, 3, 1989, pp. 493-530.

[5] Cantu-Paz E A, A summary of research on parallel genetic algorithms, IlliGAL Report No. 95007, 1995.

[6] Eshelman L J, The CHC adaptive search algorithm: How to have safe search when engaging in noon-traditional genetic recombination, In: Foundations of Genetic Algorithms , Morgan Kaufmann Publishers, 1991, pp. 265-283.

[7] Srinivas M, Patnaik L M, Adaptive probabilities of crossover and mutations in GAs, In: IEEE Trans. on SMC, 24, 4, 1994, pp. 656667.

[8] Houck C R, Joines J A, A genetic algorithm for function optimization: A MATLAB implementation, NC-SU-IE TR95-09, 1995.

[9] Tsujimura Y, Gen M, Genetic algorithms for solving multi-processor scheduling problems, In: Simulated Evolution and Learning, First Asia-Pacific Conference, SEAL'96, Taejon, Korea, Springer, 1996, pp. 106-115.

[10] Nakano R, Conventional genetic algorithm for job shop problems, In: Proceeding of the Fourth International Conference on Genetic Algorithms, 1991, pp. 474-479.

[11] Li Y, Ng K C, Uniform approach to modelbased fuzzy control system design and structural optimization, In: Genetic Algorithms and Soft Computing, Herrera $\mathrm{F}$ and Verdegay J(ed), Physica Verlag, 1996, pp. 251-278.

\section{Creative Commons Attribution License 4.0 (Attribution 4.0 International, CC BY 4.0)}

This article is published under the terms of the Creative Commons Attribution License 4.0

https://creativecommons.org/licenses/by/4.0/deed.en US 